\title{
Elective surfactant increased median duration of mechanical ventilation in preterm infants with mild to moderate respiratory distress syndrome
}

Escobedo MB, Gunkel JH, Kennedy KA, et al. Early surfactant for neonates with mild to moderate respiratory distress syndrome: a multicenter, randomized trial. J Pediatr 2004;144:804-8.

\section{$Q$ What is the efficacy of elective brief intubation for early single dose surfactant administration compared with administration as clinically indicated for preterm infants with mild to moderate respiratory distress syndrome (RDS) not requiring mechanical ventilation?}

\section{METHODS}

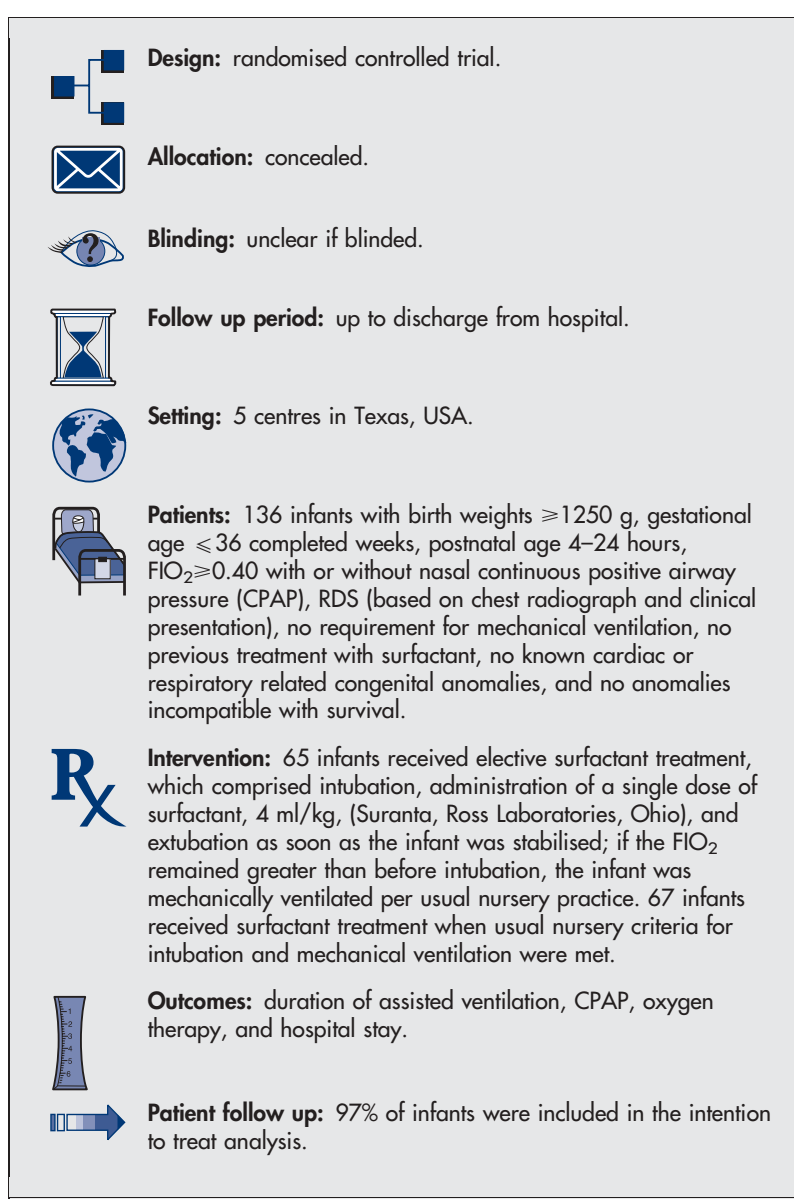

\section{MAIN RESULTS}

Median duration of assisted ventilation was longer in the elective treatment group than in the control group $(2.2 \vee 0 \mathrm{~h}, \mathrm{p}=0.001)$. However, infants in the elective treatment group were less likely to require subsequent mechanical ventilation for worsening respiratory disease (table). The groups did not differ for median duration of CPAP $(46 \vee 34 \mathrm{~h}, \mathrm{p}=0.81)$, oxygen therapy $(4.3 \vee 4.7 \mathrm{~h}, \mathrm{p}=0.20)$, or hospital stay $(20 \vee 19 \mathrm{~d}, \mathrm{p}=0.89)$.

For correspondence: Professor M B Escobedo, Department of Pediatrics, University of Oklahoma Health Sciences Center, Oklahoma City, OK, USA. marilyn-escobedo@ouhsc.edu

Source of funding: in part, Abbott Laboratories.

\section{CONCLUSIONS}

In preterm infants with mild to moderate respiratory distress syndrome not requiring mechanical ventilation, elective brief intubation for early single dose surfactant administration resulted in a slightly longer median duration of ventilation compared with administration as clinically indicated, but it slightly reduced the risk of subsequent ventilation for worsening respiratory disease. The groups did not differ for median duration of CPAP, oxygen therapy, or hospital stay.

Elective surfactant $v$ surfactant when clinically indicated (control) in preterm infants with mild to moderate respiratory distress syndrome*

\begin{tabular}{lllll}
\hline $\begin{array}{l}\text { Outcomes } \\
\text { (up to discharge) }\end{array}$ & $\begin{array}{l}\text { Elective } \\
\text { surfactant }\end{array}$ & $\begin{array}{l}\text { RRR } \\
\text { Control }\end{array}$ & $\begin{array}{l}\text { (95\% CI) } \\
\text { NNT (CI) }\end{array}$ \\
\hline $\begin{array}{l}\text { Subsequent mechanical } \\
\text { ventilation for worsening }\end{array}$ & $26 \%$ & $43 \%$ & $40 \%$ & 6 \\
respiratory disease & & & (2 to 63) & (3 to 123) \\
\hline
\end{tabular}

*Abbreviations defined in glossary; RRR, NNT, and Cl calculated from data in article.

\section{Commentary}

xogenous surfactant administration is possibly the most researched treatment available to premature infants. The study by Escobedo ef al adds to this body of knowledge because it focuses on the intubation and delivery of surfactant to more mature infants who may be at risk of RDS. These infants are not often intubated for respiratory distress but receive CPAP delivered by nasal prongs (NCPAP). The protocol for intubation of study infants was not described. This information would be valuable because the use of sedation, with or without paralysis during the procedure, may affect time to extubation and NCPAP. Furthermore, the criteria for treatment of control group infants with surfactant were not described, and this may reduce the transferability of the results to other patient populations.

The findings of this study are relevant to neonatal nurses who work in level III intensive care units because infants with RDS who do not meet clinical criteria for surfactant therapy still require expert application, maintenance, and monitoring of NCPAP. Neonatal nurse practitioners will find this information useful as they seek to choose the best RDS treatment for infants of all gestational ages. The findings are also relevant to all practitioners in neonatal units where NCPAP is used, but when surfactant and invasive ventilation are not readily available, there may be no advantage to early transport of these infants for surfactant therapy.

The results illuminate the problem of applying a treatment that is appropriate for one population to another. In view of the potential hazards of intubation, larger and more mature infants with RDS may not benefit from surfactant administration before it is clinically indicated. Development and implementation of evidence-based, standardised guidelines for the administration of surfactant should be a priority for all practitioners who care for infants with RDS.

Bonnie Rich, RN, MSc, ACNP Neonatal Intensive Care Unit, McMaster Children's Hospital Hamilton, Ontario, Canada 